\title{
Antitumor and anti-angiogenic activity of the recombinant human disintegrin domain of A disintegrin and metalloproteinase 15
}

\author{
YING HOU $^{1 *}$, MIN CHU $^{2 *}$, YANFEI CAI ${ }^{1}$, JIANYONG LEI $^{1}$, YUN CHEN $^{1}$, \\ RUIYU ZHU ${ }^{1}$, XIAOHAI GONG ${ }^{1}$, XIN MA ${ }^{1}$ and JIAN JIN ${ }^{1}$ \\ ${ }^{1}$ Laboratory of Molecular Pharmacology and Drug Design, School of Pharmaceutical Sciences; \\ ${ }^{2}$ Wuxi Medical School, Jiangnan University, Wuxi, Jiangsu 214122, P.R. China
}

Received May 28, 2014; Accepted March 4, 2015

DOI: $10.3892 / \mathrm{mmr} .2015 .3636$

\begin{abstract}
A disintegrin and metalloproteinases (ADAMs), a family of transmembrane glycoproteins, are expressed in numerous tissues and organs, and have been implicated in a variety of physiological and pathological processes. ADAM15 is unique among the ADAMs in having an Arg-Gly-Asp motif in its disintegrin domain. In the present study, the antitumor and anti-angiogenic effects of the recombinant human disintegrin domain (rhdd) ADAM15, expressed by Escherichia coli, were evaluated. rhddADAM15 inhibited the proliferation and migration of several tumor cells, with a half maximal inhibitory concentration of 1.0-6.0 $\mu \mathrm{M}$. In addition, rhddADAM15 inhibited the proliferation of Bel-7402 cells via the mitogen-activated protein kinase pathway and reduced the activation of Src. rhddADAM15 (1-10 $\mu \mathrm{M})$ inhibited the proliferation, migration and tube formation of vascular endothelial EA.hy926 cells. $\mathrm{G}_{0} / \mathrm{G}_{1}$ arrest $(10.96 \pm 1.40 \%)$ and apoptotic cells $(55.85 \pm 1.06 \%)$ were observed in the EA.hy926 cells treated with $4 \mu \mathrm{M}$ and $6 \mu \mathrm{M}$ rhddADAM15, respectively. In vivo, rhddADAM15 significantly inhibited angiogenesis in zebrafish. rhddADAM15 at concentrations of $20 \mathrm{nmol} / \mathrm{fish}$ or $5 \mathrm{nmol} /$ fish inhibited the angiogenesis of subintestinal and intersegmental vessels in the zebrafish by $72 \pm 1.26$ and $48 \pm 2.92 \%$, respectively. In conclusion, the results of the present study identified rhddADAM15 as a potent inhibitor of tumor formation and angiogenesis, rendering it a promising tool for use in anticancer treatment.
\end{abstract}

Correspondence to: Professor Jian Jin, Laboratory of Molecular Pharmacology and Drug Design, School of Pharmaceutical Sciences, Jiangnan University, 1800 Lihu Road, Wuxi, Jiangsu 214122, P.R. China

E-mail: jinjian31@126.com

${ }^{*}$ Contributed equally

Key words: A disintegrin and metalloproteinase 15, disintegrin, Src, angiogenesis, zebrafish

\section{Introduction}

A disintegrin and metalloproteinases (ADAMs) are a family of transmembrane proteins, anchored to the cell membrane surface (1), which are implicated in a variety of physiological and pathological processes, including sperm-egg binding, neuronal development and myotube formation (2). In addition, ADAMs have been observed to be overexpressed in a variety of types of tumor (3).

Human ADAM15 is unique among the ADAM family, containing the integrin binding motif Arg-Gly-Asp (RGD) sequence in its disintegrin domain (4). ADAM15 is important in cell adhesion, extracellular matrix degradation, intracellular signal transduction and pathological changes in tumors. Previous studies have revealed that the disintegrin domain of ADAM15 interacts with integrins $\alpha v \beta 3, \alpha 5 \beta 1$ and $\alpha 9 \beta 1$, and inhibits platelet aggregation and cell migration $(3,5,6)$. The downregulation or overexpression of ADAM15 alters cell-cell interactions and cell behavior (6-8). In addition, ADAM15 is overexpressed in several types of solid tumor and is closely associated with tumor occurrence and development of $(9,10)$.

Angiogenesis is essential in the process of tumor growth. Certain proteolytic fragments of the extracellular matrix, including endostatin and angiostatin have been found to inhibit tumor angiogenesis $(11,12)$. Previous studies have observed that ADAM15 is overexpressed in the vascular endothelial cells of tumors $(13,14)$. In a previous investigation using a mouse model of retinopathy of prematurity, neovascularization in ADAM $15^{-/-}$mice was markedly reduced compared with wild-type mice. In addition, the tumor sizes in the ADAM $15^{-/}$mice were significantly smaller than those in the wild-type mice (15). Trochon-Joseph et al (10) found that the recombinant disintegrin domain of ADAM15 inhibits in vitro angiogenesis and the growth of breast and lung cancer metastases in melanoma.

In our previous investigations, the recombinant human disintegrin domain of ADAM15 (rhddADAM15) was expressed in Escherichia coli, and the inhibitory activity of rhddADAM15 on Bel-7402 liver cancer cells was evaluated $(16,17)$. The present study aimed to assess the antitumor and anti-angiogenic activities of rhddADAM15 in vivo, using a zebrafish model. The human umbilical vein cell line EAhy926, which is an adherent cell line and was established 
by fusing primary human umbilical vein cells with a thioguanine-resistant clone of A549, was also used. The mechanism underlying the inhibition of proliferation of the Bel-7402 cells was investigated, and the inhibitory effects of rhddADAM15 on vascular endothelial cells were evaluated, to determine whether rhddADAM15 affects angiogenesis in zebrafish.

\section{Materials and methods}

Cell culture. Human breast cancer MCF-7, mouse melanoma B16, human cervical cancer Hela, human pancreatic cancer 8988, human liver cancer Bel-7402 and human liver cancer HEPG-2 cells were all purchased from the Cell Bank of the Chinese Academy of Sciences (Shanghai, China), and doxorubicin resistant breast cancer MCF-7/ADM and taxol resistant breast cancer MCF-7/PTX cells were produced in our laboratory. All of the above cells were grown in RPMI 1640 essential medium (Gibco Life Technologies, Carlsbad, CA, USA), supplemented with $10 \%$ fetal bovine serum (Gibco Life Technologies) and penicillin-streptomycin $(100 \mathrm{U} / \mathrm{ml}$ and $100 \mu \mathrm{g} / \mathrm{ml}$, respectively; Gibco Life Technologies). The cells were incubated in $5 \% \mathrm{CO}_{2}$ at $37^{\circ} \mathrm{C}$. Technical support for the zebrafish study was provided by Hunter Biotechnology, Inc. (Hangzhou, China) and was approved by the ethics committee of the Association for Assessment and Accreditation of Laboratory Animal Care International (certificate no. 001458) and Science and Technology Department of Zhejiang Province, China (certificate no. SYXK20120171).

Cell proliferation assay. A sulforhodamine B assay (SRB; Sigma-Aldrich, St. Louis, MO, USA) was used to assess cell proliferation. The cells were seeded into a 96-well plate (Corning Life Sciences, Shanghai, China) at a density of $6-7 \times 10^{3}$ cells/well and cultured for $24 \mathrm{~h}$ at $37^{\circ} \mathrm{C}$. Subsequently, the medium was removed and replaced with $100 \mu \mathrm{l}$ medium containing rhddADAM15 (produced in our laboratory; 0, 1.0, $1.5,2.0,4.0,5.0,8.0$ and $10.0 \mu \mathrm{mol} / \mathrm{l}$ ). Following incubation for $24 \mathrm{~h}$, the medium was removed and the cells were fixed by adding $100 \mu \mathrm{l}$ cold $10 \%$ trichloroacetic acid (Sigma-Aldrich) and incubated for $60 \mathrm{~min}$ at $4^{\circ} \mathrm{C}$. The fixed cells were then washed in water and stained with $100 \mu 10.4 \%$ (w/v) SRB at $37^{\circ} \mathrm{C}$. After $30 \mathrm{~min}$, the unbound SRB was washed off with $1 \%$ acetic acid (Sinopharm Chemical Reagent Co., Ltd., Shanghai, China), and the stained cells were solubilized with $100 \mu 110$ mM Tris-base (Sinopharm Chemical Reagent Co., Ltd.). The absorbance of the stained cells in the wells was measured at $540 \mathrm{~nm}\left(\mathrm{Abs}_{540 \mathrm{~nm}}\right)$ using a Multiskan MK2 microplate reader (Thermo Fisher Scientific, Waltham, MA, USA). The inhibitory rate of cell proliferation was calculated using the following formula: Inhibitory rate = $\left(\mathrm{Abs}_{540 \mathrm{~nm}, \text { control }}-\mathrm{Abs}_{540 \mathrm{~nm}, \text { rhddADAM15}}\right) /\left(\mathrm{Abs}_{540 \mathrm{~nm}, \mathrm{control}}-\mathrm{Abs}_{540 \mathrm{~nm}}\right.$ blank) x $100 \%$.

Cell migration assay. A wound-healing assay was used to assess cell migration. The cells were seeded into a 24 -well plate at a density of $1-5 \times 10^{5} /$ well, and were incubated for $24 \mathrm{~h}$ at $37^{\circ} \mathrm{C}$. A 'wound' was formed by manually scraping the monolayer in the middle of each well with a pipette tip. The floating cells were washed off with phosphate-buffered saline
(PBS) and the first set of images of each well were captured. The location of each image was marked on the bottom of each well. Fresh medium, or medium with rhddADAM15 (0, 1.0, $1.5,2.0,4.0$ and $8.0 \mu \mathrm{mol} / \mathrm{l})$, was added and the cells were incubated for a further $24 \mathrm{~h}$. Subsequently, a second set of images were captured at the marked locations. The area of the wound (A), indicating the ability of the cells to migrate, was measured using ImageJ software, version 1.4.3.67 (National Institutes of Health, Bethesda, MD, USA). The inhibitory rate of cell migration was calculated using the following formula: Rate of migration $(\mathrm{MR})=\left(\mathrm{A}_{0 \mathrm{~h}}-\mathrm{A}_{24 \mathrm{~h}}\right) / \mathrm{A}_{0 \mathrm{~h}} \times 100 \%$. The rate of inhibition of migration was calculated as: $\mathrm{MR}_{\text {control }}-\mathrm{MR}_{\text {rhddADAM15 }}$.

Western blot analysis. Following treatment with rhddADAM15, the cells were collected, lysed (Beyotime Institute of Biotechnology, Nantong, China) and clarified by centrifugation (5418; Eppendorf, Hamburg, Germany) for $10 \mathrm{~min}$ at $4^{\circ} \mathrm{C}$, $12,391 \mathrm{x}$ g. Subsequently, the samples were incubated with an equal volume of $2 \mathrm{X}$ sodium dodecyl sulfate (SDS; Sinopharm Chemical Reagent Co., Ltd.) sample buffer and heated for $5 \mathrm{~min}$ at $95^{\circ} \mathrm{C}$. SDS-PAGE was then performed, following which the proteins were transferred onto nitrocellulose membranes (Pall Life Sciences, Ann Arbor, MI, USA). The membranes were blocked with $5 \%$ bovine serum albumin in Tris-buffered saline- $0.1 \%$ Tween 20 for $1 \mathrm{~h}$ at room temperature. The blots were then incubated with the following antibodies for $\sim 2 \mathrm{~h}$ at $4^{\circ} \mathrm{C}$ : Rabbit polyclonal phosphorylated Src Y416 (\#2101; 1:1,000; Cell Signaling Technology, Boston, MA, USA), rabbit polyclonal phosphorylated Src Y527 (\#2105; 1:1,000; Cell Signaling Technology), mouse monoclonal total Src (ab16885; 1:100; Abcam, Cambridge, UK) and mouse monoclonal $\beta$-actin (AA128; 1:1,000; Beyotime Institute of Biotechnology). The blots were then incubated with horseradish peroxidase-conjugated goat anti-rabbit (A0208) and goat anti-mouse (A0216) IgG secondary antibodies (Beyotime Institute of Biotechnology) for $1 \mathrm{~h}$ at $37^{\circ} \mathrm{C}$. The blots were visualized using enhanced chemiluminescence reagents (Beyotime Institute of Biotechnology). $\beta$-actin was used as the internal reference.

Tube formation assay. Matrigel basement membrane matrix (Matrigel; BD Biosciences, Franklin Lakes, NJ, USA) was thawed and 96-well plates and tips were precooled at $4^{\circ} \mathrm{C}$ overnight. Subsequently, the plates were coated with $60 \mu \mathrm{l}$ Matrigel and incubated for $30 \mathrm{~min}$ at $37^{\circ} \mathrm{C}$. The EAhy926 cells were seeded at a density of $6-8 \times 10^{4} /$ well with different concentrations of rhddADAM15 $(0,1.0,2.0,4.0,6.0$ and $8.0 \mu \mathrm{mol} / \mathrm{l})$. After $6 \mathrm{~h}$ incubation at $37^{\circ} \mathrm{C}$, the tube forming structures were observed and images were captured under a microscope (CKX41; Olympus, Tokyo, Japan). At least three images were captured randomly in each group and the number of formed tubes were counted.

Analysis of apoptosis. An Annexin V-Propidium Iodide (PI) Apoptosis Detection kit (Beyotime Institute of Biotechnology) was used to detect the apoptosis induced by rhddADAM15. The EAhy926 cells were seeded at a density of $3-5 \times 10^{5} /$ well in 6 -well plates and cultured overnight, followed by treatment with different concentrations of rhddADAM15 $(0,4.0$ and $6.0 \mu \mathrm{mol} / \mathrm{l})$ for $6 \mathrm{~h}$ at $37^{\circ} \mathrm{C}$. The cells were then 
Table I. Inhibitory effect of recombinant human disintegrin domain A disintegrin and metalloproteinase 15 on the proliferation and migration of tumor cell lines.

Cell line $\mathrm{IC}_{50}$ of proliferation $(\mu \mathrm{mol} / \mathrm{l})$ $\mathrm{IC}_{50}$ of migration $(\mu \mathrm{mol} / \mathrm{l})$

MCF-7 human breast cancer

2.90

5.30

5.77

3.60

2.44

5.76

1.14

4.73
1.95

2.19

3.86

1.64

0.61

4.73

2.09

1.98

$\mathrm{IC}_{50}$, half maximal inhibitory concentration.

harvested and washed twice with PBS, followed by resuspension in binding buffer and staining with annexin $\mathrm{V}$ to detect early apoptotic cells for $10 \mathrm{~min}$. The annexin $\mathrm{V}$ was removed via centrifugation at $1,000 \mathrm{x} \mathrm{g}$ for $5 \mathrm{~min}$ at room temperature, and the cells were washed and stained with PI, to detect early and late apoptotic cells, for $5 \mathrm{~min}$ at room temperature. The percentage of apoptotic cells was determined using flow cytometry (BD Biosciences) and the data were processed using Cell Quest software, version 6.0 (BD Biosciences).

Cell cycle analysis. PI staining was used to analyze the cell cycle. The cells were seeded at a density of $3-5 \times 10^{5} /$ well in a 6 -well plate and cultured overnight, followed by treatment with or without rhddADAM 15 for $24 \mathrm{~h}$ at $37^{\circ} \mathrm{C}$. Subsequently, the cells were harvested, washed twice with PBS and fixed with $70 \%$ cold ethanol for at least $2 \mathrm{~h}$ at $4^{\circ} \mathrm{C}$. The cells were then washed and incubated with $50 \mu \mathrm{g} / \mathrm{ml}$ RNase (Sigma-Aldrich) and stained using $10 \mu \mathrm{g} / \mathrm{ml}$ PI (Beyotime Institute of Biotechnology) at $37^{\circ} \mathrm{C}$ for $30 \mathrm{~min}$. The cell cycle was then evaluated by flow cytometry and the data were analyzed using Modfit software (Verity Software House, Topsham, ME, USA).

Angiogenesis model in zebrafish. The flila: enhanced green fluorescent protein (EGFP) transgenic zebrafish used in the present study were raised and maintained according to the guidelines of the ethics committees mentioned at Wenzhou Medical University (Wenzhou, China), and the zebrafish embryos were generated by natural pair-wise mating in a light:dark cycle of 14:10 h/day (18). The zebrafish were fed with Artemia and cultured in water containing sea salt $(200 \mathrm{mg} / \mathrm{l})$ at $\sim \mathrm{pH} 7.2$ and $28^{\circ} \mathrm{C}$ in an experimental tank system made of polycarbonate. The present study was approved by the Association for Assessment and Accreditation of Laboratory Animal Care (001458) and the Science and Technology Department of Zhejiang Province, China (SYXK20120171). The subintestinal vessels (SIVs) and intersegmental vessels (ISVs) of the flila:EGFP transgenic zebrafish embryos were observed to evaluate the anti-angiogenic activity of rhddADAM15. To assess the anti-SIV and anti-ISV angiogenic activity of rhddADAM15, flila:EGFP transgenic zebrafish embryos were used at $48 \mathrm{~h}$ post-fertilization (hpf) and $23 \mathrm{hpf}$, respectively. At least 10 zebrafish embryos, allocated randomly, were used in each experimental group, including a control group. Either rhddADAM15, positive control (endostatin; Simcere Pharmaceutical, Nanjing, China) or PBS was microinjected into the yolk-sac of the flila:EGFP transgenic zebrafish embryos. Images of the zebrafish embryos in each group were captured at 0,6,12 and $24 \mathrm{~h}$ post-injection, to evaluate the time course of rhddADAM15 onset and to assess the area (AS) of the SIVs and the pairs (P) of the ISVs, which are indicative of the angiogenic ability of zebrafish. The inhibitory rates of angiogenesis of the SIVs or ISVs were calculated using the following formulae: Inhibitory rate of SIVs $(\%)=\left(1-\mathrm{AS}_{\text {rhddADAM15 }} / \mathrm{AS}_{\text {control }}\right) \times 1$ $00 \%$ and, inhibition rate of ISVs $(\%)=\left(1-\mathrm{P}_{\text {rhddADAM } 15} / \mathrm{P}_{\text {cont }}\right.$ rol) $\mathrm{x} 100 \%$.

Statistical analysis. The results are presented as the mean \pm standard error of the mean of triplicates from at least three independent experiments. Statistical differences were determined using Dunnett's t-test with GraphPad Prism software, version 5.01 (GraphPad Software, Inc., La Jolla, CA, USA). $\mathrm{P}<0.05$ was considered to indicate a statistically significant difference.

\section{Results}

rhddADAM15 inhibits the proliferation andmigration of tumor cell lines in vitro. The SRB assay revealed that rhddADAM15 inhibited the proliferation of several tumor cell lines, with a half maximal inhibitory concentration $\left(\mathrm{IC}_{50}\right)$ range between 1.04 and $5.77 \mu \mathrm{M}$ (Table I). The most sensitive cells were the Bel-7402 human liver cancer cells, with an $\mathrm{IC}_{50}$ of $1.04 \mu \mathrm{M}$. rhddADAM15 also inhibited the migration of the eight tumor cell lines, which was determined using a wound-healing assay, the most sensitive of which were the Bel-7402 cells, with an $\mathrm{IC}_{50}$ of $2.09 \mu \mathrm{M}$ (Table I).

rhddADAM15 reduces Src activation in Bel-7402 cells. It has been demonstrated that rhddADAM15 inhibits the proliferation and migration of Bel-7402 cells in vitro and in vivo, and induces apoptosis and partial $\mathrm{G}_{2} / \mathrm{M}$ phase arrest (17). To investigate the mechanism underlying the inhibition 
A

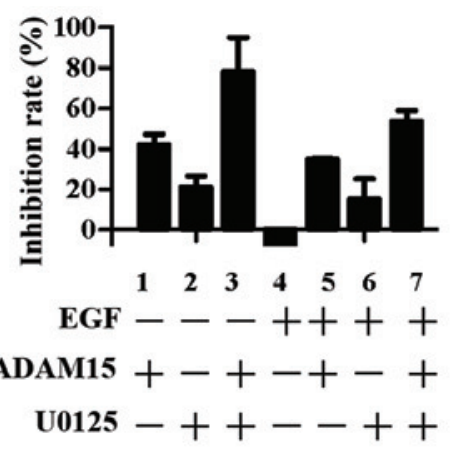

B

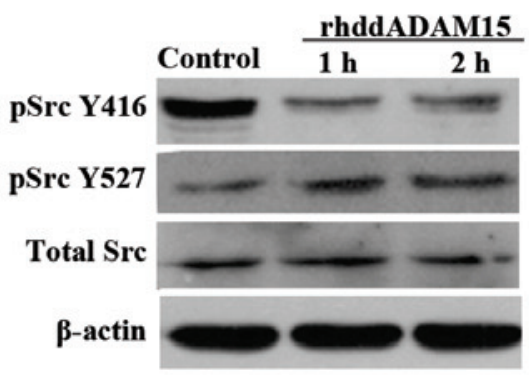

C

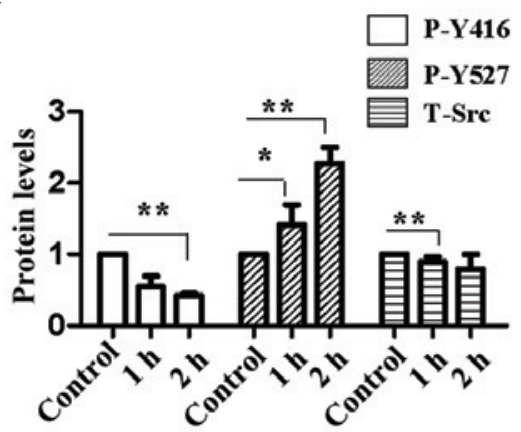

Figure 1. rhddADAM15 reduces Src activation in Bel-7402 cells. (A) Effects of rhddADAM15, EGF and U025 on the proliferation of Bel-7402 cells. ' + ' indicates presence of reagent and '-' indicates absence of reagent. (B) Changes in the levels of Src and pSrc in Bel-7402 cells following treatment with rhddADAM15. Following treatment of the Bel-7402 cells with rhddADAM15 for 1 or $2 \mathrm{~h}$, the levels of total Src, and pSrc Y416 and Y529 were detected using western blot analysis. (C) Quantification of $\mathrm{B}\left({ }^{*} \mathrm{P}<0.05\right.$ and ${ }^{* *} \mathrm{P}<0.01$, vs. untreated control). Data are expressed as the mean \pm standard error of the mean. rhdd, recombinant human disintegrin domain; EGF, epidermal growth factor; ADAM15, A disintegrin and metalloproteinase 15; p, phosphorylated.

A

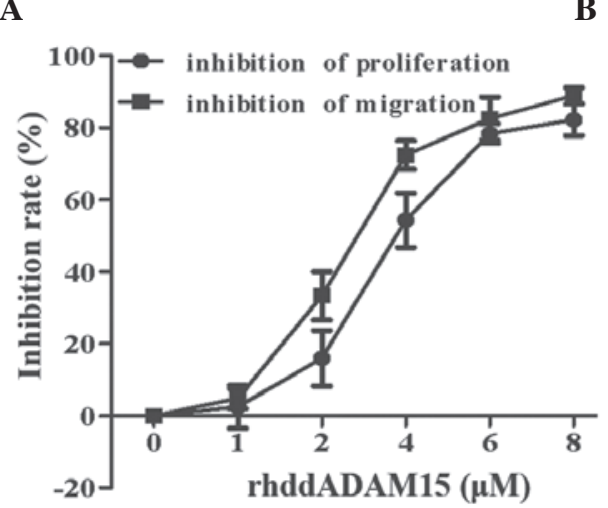

B

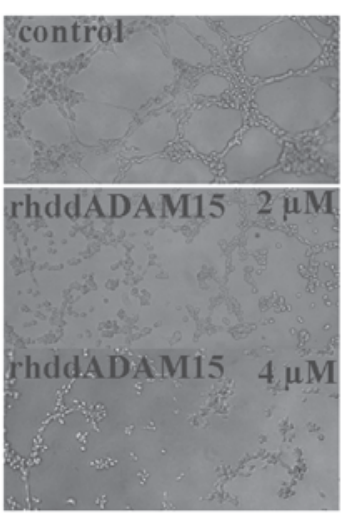

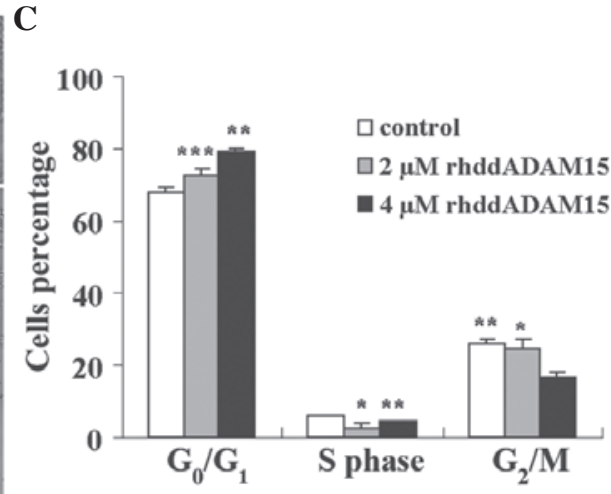

rhddADAM15

D

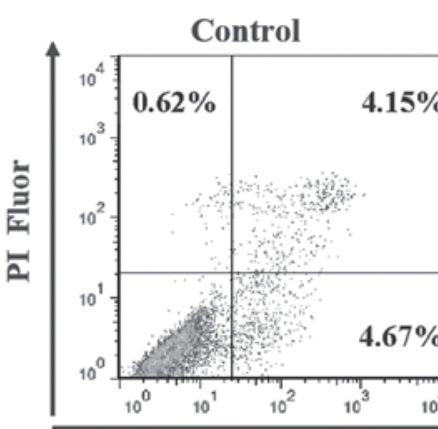

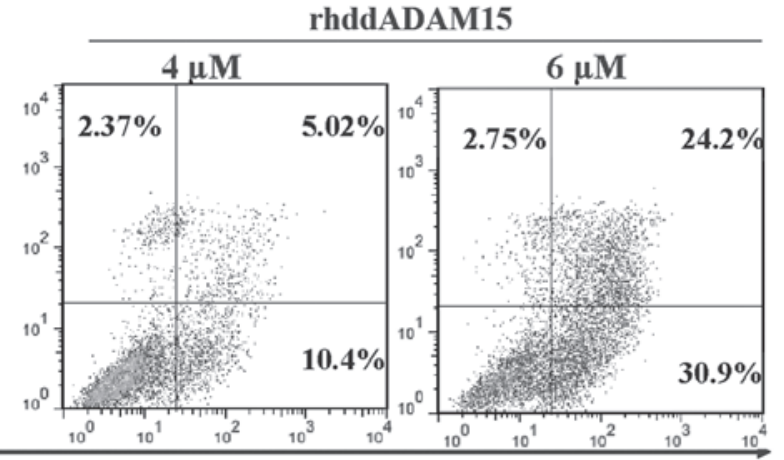

Annexin V-FITC Fluor

Figure 2. rhddADAM15 inhibits the proliferation, migration and tube formation, and induces cell cycle arrest and apoptosis of Eahy926 cells. (A) Inhibitory effect of rhddADAM15 on the proliferation and migration of EAhy926 cells. (B) Representative images of tube formation in the EAhy926 cells following rhddADAM15 treatment. Cells were analyzed under a fluorescence microscope (magnification, x40). (C) Assessment of EAhy926 cell cycle following rhddADAM15 treatment. (D) Assessment of apoptosis in EAhy926 cells treated with rhddADAM15. In the four-quadrant plots, normal cells are in the lower-left, necrotic cells are in the upper-right, early apoptotic cells are in the lower-right, and late apoptotic cells in are the upper-right. Data are expressed as the mean \pm standard error of the mean $\left(\mathrm{n}=3 ;{ }^{*} \mathrm{P}<0.0,{ }^{* *} \mathrm{P}<0.01\right.$ and ${ }^{* * * *} \mathrm{P}<0.001$, vs. untreated control). rhdd, recombinant human disintegrin domain; ADAM15, A disintegrin and metalloproteinase 15; FITC, fluorescein isothiocyanate; PI, propidium iodide.

of proliferation, the present study investigated whether rhddADAM15 affected the mitogen-activated protein kinase (MAPK) signaling pathway, one of the most important pathways associated with cell proliferation and migration. U0125, an inhibitor of extracellular signal regulated kinase (Erk)1/2, and epidermal growth factor (EGF), an agonist of MAPK, were used in the present study. The concentration of rhddADAM15, EGF and U0125 were 1.0, 0.8 and $10 \mathrm{nM}$, respectively. As shown in Fig. 1A, rhddADAM15 inhibited the proliferation of the Bel-7402 cells, with an inhibitory rate of $41.9 \%$ (column 1). Similarly, U0125 inhibited the proliferation of the Bel-7402 cells, and the inhibitory effect was increased when combined with rhddADAM15 (columns 2 and 3). By contrast, EGF increased the proliferative effect 
A
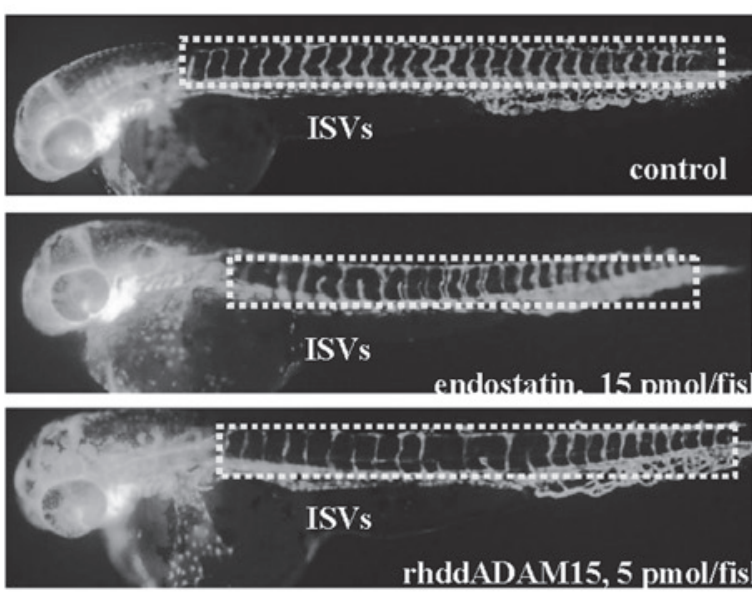

C
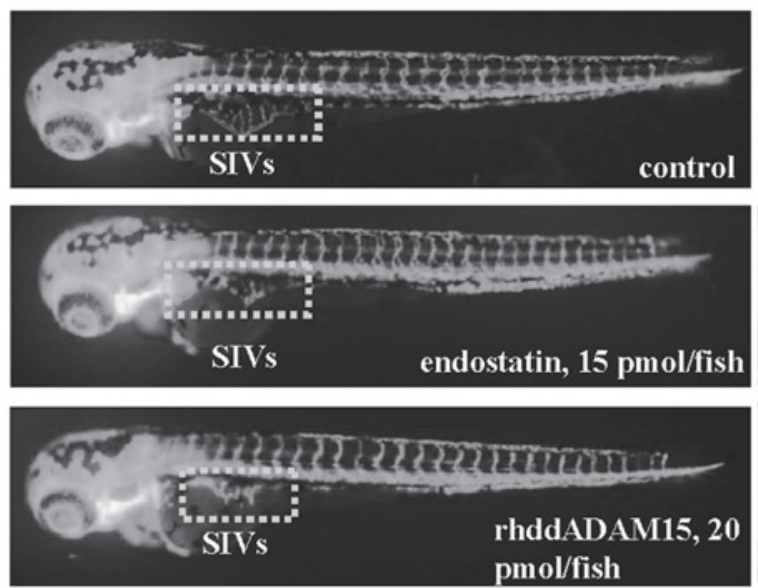
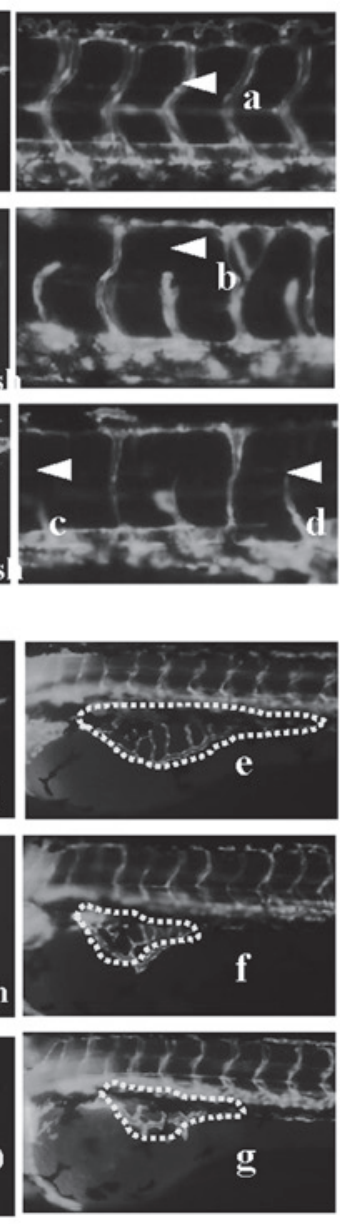

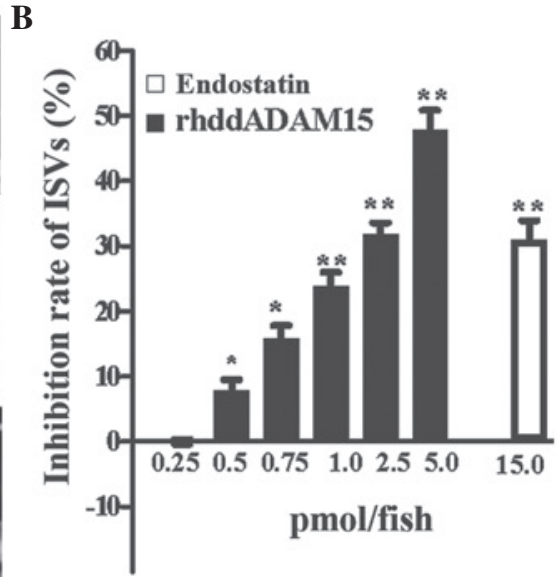

D

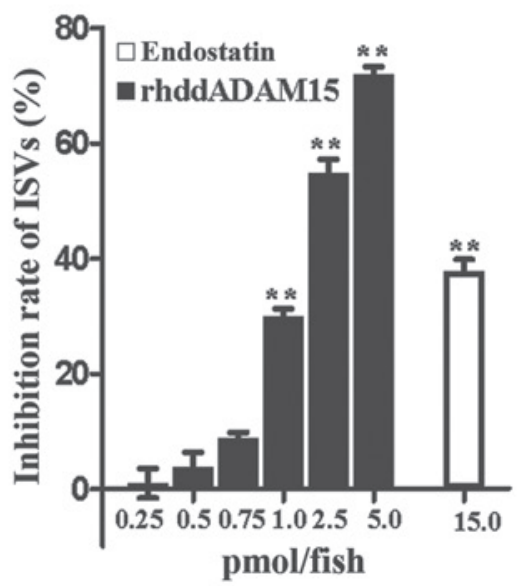

Figure 3. rhddADAM15 inhibits ISV and SIV angiogenesis in zebrafish. (A) Representative images of ISV angiogenesis in zebrafish embryos following treatment with rhddADAM15. Dotted lines indicate the ISVs in the zebrafish embryos. White arrowheads indicate (a) normal vessels in the control group and the (b and c) absence and (d) random thinning of vessels following treatment with endostatin or rhddADAM15. (B) Inhibitory rates of ISV angiogenesis in zebrafish following treatment with rhddADAM15. (C) Representative images of SIV angiogenesis in zebrafish embryos following treatment. The border of the SIVs (dotted lines) in the (e) control group was regular. Following treatment with (f) endostatin or (g) rhddADAM15, the border was irregular and the area was reduced. (D) Inhibitory rates of SIV angiogenesis in zebrafish following rhddADAM15 treatment. Data are expressed as the mean \pm standard error of the mean $\left(\mathrm{n}=10 ;{ }^{*} \mathrm{P}<0.05\right.$ and ${ }^{* *} \mathrm{P}<0.01$ vs. control). rhdd, recombinant human disintegrin domain; ADAM15, A disintegrin and metalloproteinase 15; SIV, subintestinal vessels; ISV, intersegmental vessels.

(column 4) and weakened the inhibitory effect, partially induced by rhddADAM15 or U025 (columns 5-7). Src is an integrator of divergent signals and channels phosphorylation signals through the Ras/Raf/ERK1/2 signaling pathway and, in certain cells, the phosphatidylinositol 3-kinase/AKT signaling pathway (19). To investigate whether rhddADAM15 affected the activation of Src, the phosphorylation levels of Src at Tyrosine 416 (Y416) and Tyrosine 529 (Y529) of the Bel-7402 cells were evaluated, following treatment with rhddADAM15. As shown in Fig. 1B, no significant difference was observed in the total level of Src in the Bel-7402 cells treated with or without rhddADAM15. Following treatment with rhddADAM15, a significant reduction in the phosphorylation levels of Y416 was detected in the Bel-7402 cells, compared with the control cells. By contrast, the phosphorylation levels of Y527 was increased (Fig. 1B). The dephosphorylation of Src Y529 is a requirement for Src activation. The present data revealed that rhddADAM15 inhibited the phosphorylation of Y416 and dephosphorylation of Y529, therefore, rhddADAM15 downregulated the activation of Src.
rhddADAM15 inhibits the proliferation, migration and tube formation of EAhy926 cells. To investigate the effect of rhddADAM15 on angiogenesis, its activity on the proliferation, migration and tube formation of EAhy926 cells was evaluated. rhddADAM15 inhibited the proliferation and migration of EAhy926 cells in a dose-dependent manner, and the $\mathrm{IC}_{50}$ values were 3.78 and $3.18 \mu \mathrm{M}$, respectively (Fig. 2A). The EAhy926 cells formed tube structures on the Matrigel following $6 \mathrm{~h}$ incubation (Fig. 2B). Although the cells remained adhered to each other, few tubes were identified in each image, and the number of tubes formed was $79 \%$ lower following rhddADAM15 treatment at a concentration of $4 \mu \mathrm{M}$ (Fig. 2B). This result demonstrated that rhddADAM15 inhibited the formation of tube structures.

The cell cycle and apoptotic rates were analyzed to determine how rhddADAM15 inhibited the proliferation of EAhy 926 cells. The number of cells in the $\mathrm{G}_{0} / \mathrm{G}_{1}$ phase increased and the number in the $G_{2}$ and $S$ phase decreased, indicating that a proportion of the cells were arrested at $G_{0} / G_{1}$ (Fig. 2C). The annexin V-PI double-staining method was used to determine the percentage of apoptotic cells after $6 \mathrm{~h}$ rhddADAM15 
treatment. rhddADAM15 induced apoptosis in the EAhy926 cells in a dose-dependent manner, with a maximum inhibitory rate of $55.1 \pm 2.3 \%$ at $6 \mu \mathrm{M}$ rhddADAM15 (Fig. 2D), which was a 7-fold increase compared with the controls $(\sim 8.8 \%)$, suggesting that rhddADAM15 markedly induced apoptosis in the EAhy926 cells.

rhddADAM15 inhibits angiogenesis in zebrafish. rhddADAM15-induced anti-angiogenic activity was evaluated in the ISVs and SIVs of zebrafish embryos. A total of 28 pairs of well-arranged ISVs in zebrafish embryos were observed in the fluorescent-labeled vascular endothelial cells (Fig. 3A). Following treatment with rhddADAM15 or endostatin for $24 \mathrm{~h}$, the zebrafish embryos exhibited a decreased in ISV formation (Fig. 3A-D). Compared with the negative control, the zebrafish embryos treated with rhddADAM15 exhibited a thinning or absence of ISVs, in a dose-dependent manner, with a maximum inhibitory rate of $48 \pm 2.92 \%$ at $5.0 \mathrm{pmol} /$ fish rhddADAM15.

The anti-angiogenic activity of rhddADAM15 on SIVs was also assessed, which formed a regular basket-like network (Fig. 3C and E). Following treatment with rhddADAM15 or endostatin, the SIVs were irregular and the area was reduced in a dose-dependent manner (Fig. 3C, F and G). The maximum inhibitory rate was $72 \pm 1.26 \%$ at $20.0 \mathrm{pmol} /$ fish rhddADAM15 and $40 \pm 1.36 \%$ at $20 \mathrm{pmol} / \mathrm{fish}$ with endostatin. In addition, as shown in Fig. 3C, the ISVs, which had formed in these zebrafish did not exhibit any significant pathological changes, indicating that rhddADAM15 or endostatin inhibited the process of angiogenesis, not the vessels that formed. These results demonstrated that rhddADAM15 caused marked inhibition of the angiogenesis of ISVs and SIVs in zebrafish, with a more marked effect compared with endostatin.

\section{Discussion}

The interaction between ECM and integrins is important in tumor progression. The disintegrin domain of human ADAM15, which contains an RGD motif, is an important functional domain and has been revealed to mediate cell-cell and cell-matrix interactions (20,21). Previous studies have demonstrated that ADAM15 is overexpressed in tumor cells and vascular endothelial cells in multiple types of tumor. However, the function of ADAM15 in tumor progression remains to be elucidated, as do the potential anti-angiogenic activities of rhddADAM15 in vivo, as an exogenous therapeutic protein.

The present study demonstrated that, as an exogenous protein, rhddADAM15 inhibited the proliferation and migration of several tumor cell lines in vitro. It was observed in our previous study that rhddADAM15 induces apoptosis via the caspase 8 and caspase 9 pathways (17). Since rhddADAM15 is considered to interact with integrins $(5,6,22)$, the signaling pathways associated with integrins and cell proliferation were investigated. The results revealed that rhddADAM15 downregulates the activity of c-Src and regulates the phosphorylation of cyclin-dependent kinase 2 (data not shown). However, the specific target remains to be elucidated.

As anti-angiogenic therapies have the advantage of exhibiting broad-spectrum effects with a low risk for metastasis, there has been increasing attention on the identification of anti-angiogenic drug treatments that control tumor growth. Currently, the majority of anti-angiogenic drug treatments target vascular endothelial growth factor (VEGF), and several VEGF inhibitors have been approved for clinical use in the treatment of cancer and eye diseases $(23,24)$. However, these agents require optimization, and the development of novel targets is required for several reasons: Certain patients do not respond to the treatment, drug resistance may occur during the course of the treatment and importantly, a previous study revealed that tumor angiogenesis can occasionally become VEGF-independent (25). Certain integrins are involved in angiogenic processes, and cyclic RGD peptides have been observed to inhibit angiogenesis, leading to tumor regression via the targeting of integrins (14,26-29).

The disintegrin domain of human ADAM15 contains the RGD integrin binding motif. In addition, ADAM15 has been found to be upregulated on the angiogenic endothelial cell surface (13). Therefore, the anti-angiogenic activity of rhddADAM15 was further evaluated in the present study. Although the in vitro activity of rhddADAM15 against angiogenesis has been evaluated (10), investigation of angiogenesis in vivo faces several challenges. The use of zebrafish offers an intuitive and effective model in the investigation of in vivo angiogenesis and tumor formation (30-32) for advanced drug screening, owing to their small size, short life cycle and transparency. In the present study, rhddADAM15 inhibited the proliferation, migration and tube formation of EAhy926 cells, which was in agreement with a previous study by Trochon-Joseph et al (10). In addition, the present study demonstrated that rhddADAM15 induced apoptosis and $\mathrm{G}_{0} / \mathrm{G}_{1}$ phase arrest in the EAhy926 cells, which was responsible for the inhibition of proliferation. In addition, rhddADAM15 in the zebrafish exhibited superior anti-angiogenic activity compared with endostatin.

ADAM15 is a multi-domain and multifunctional protein, and several molecular pathways have been suggested to explain its effects. The key mechanisms of rhddADAM15 are considered to be associated with its integrin-binding domain (10). Crociani et al (33) identified a novel angiogenic pathway in colorectal cancer, which is triggered by $\beta 1$ integrin-mediated adhesion and leads to the secretion of VEGF-A. In addition, Lucena et al (34) found that the r-mojastin 1 and r-viridistatin 2 recombinant disintegrins, which bind to $\alpha v \beta 3$ and $\alpha v \beta 5$, have anti-angiogenic activities. Although the precise mechanisms underlying the anti-angiogenic activity of rhddADAM15 requires further investigation, the results of the present study demonstrated the anti-angiogenic activity of rhddADAM15 in vivo for the first time, to the best of our knowledge, and revealed that, as a recombinant exogenous protein, rhddADAM15 is effective against angiogenesis in vitro and in vivo and may be a potential anticancer agent.

\section{Acknowledgements}

The authors would like to thank Dr Li Chunqi (Wenzhou Medical University, China) for providing the flila:EGFP transgenic zebrafish and Dr IC Bruce for reading the manuscript. The present study was supported by the China National Natural Science Foundation (grant no. 30772586). 


\section{References}

1. Wolfsberg TG and White JM: ADAMs in fertilization and development. Dev Biol 180: 389-401, 1996.

2. Seals DF and Courtneidge SA: The ADAMs family of metalloproteases: multidomain proteins with multiple functions. Genes Dev 17: 7-30, 2003.

3. Arribas J, Bech-Serra JJ and Santiago-Josefat B: ADAMs, cell migration and cancer. Cancer Metastasis Rev 25: 57-68, 2006.

4. Krätzschmar J, Lum L and Blobel CP: Metargidin, a membrane-anchored metalloprotease-disintegrin protein with an RGD integrin binding sequence. J Biol Chem 271: 4593-4596, 1996.

5. Zhang XP, Kamata T, Yokoyama K, Puzon-McLaughlin W and Takada Y: Specific interaction of the recombinant disintegrin-like domain of MDC-15 (metargidin, ADAM-15) with integrin alphavbeta3. J Biol Chem 273: 7345-7350, 1998.

6. Eto K, Puzon-McLaughlin W, Sheppard D, Sehara-Fujisawa A, Zhang XP and Takada Y: RGD-independent binding of integrin alpha9betal to the ADAM-12 and -15 disintegrin domains mediates cell-cell interaction. J Biol Chem 275: 34922-34930, 2000

7. Nath D, Slocombe PM, Stephens PE, et al: Interaction of metargidin (ADAM-15) with alphavbeta3 and alpha5beta1 integrins on different haemopoietic cells. J Cell Sci 112: 579-587, 1999.

8. Herren B, Garton KJ, Coats S, Bowen-Pope DF, Ross R and Raines EW: ADAM15 overexpression in NIH3T3 cells enhances cell-cell interactions. Exp Cell Res 271: 152-160, 2001.

9. Moss ML and Bartsch JW: Therapeutic benefits from targeting of ADAM family members. Biochemistry 43: 7227-7235, 2004.

10. Trochon-Joseph V, Martel-Renoir D, Mir LM, et al: Evidence of antiangiogenic and antimetastatic activities of the recombinant disintegrin domain of metargidin. Cancer Res 64 2062-2069, 2004.

11. O'Reilly MS, Boehm T, Shing Y, et al: Endostatin: an endogenous inhibitor of angiogenesis and tumor growth. Cell 88 : 277-285, 1997.

12. O'Reilly MS, Holmgren L, Shing Y, et al: Angiostatin: a novel angiogenesis inhibitor that mediates the suppression of metastases by a Lewis lung carcinoma. Cell 79: 315-328, 1994

13. Herren B, Raines EW and Ross R: Expression of a disintegrin-like protein in cultured human vascular cells and in vivo. FASEB J 11: 173-180, 1997.

14. Brooks PC, Montgomery AM, Rosenfeld M, et al: Integrin alpha $v$ beta 3 antagonists promote tumor regression by inducing apoptosis of angiogenic blood vessels. Cell 79: 1157-1164, 1994.

15. Horiuchi K, Weskamp G, Lum L, et al: Potential role for ADAM15 in pathological neovascularization in mice. Mol Cell Biol 23: 5614-5624, 2003

16. Wu J,Zhang L, Lei J, et al: Enhancement of recombinant human ADAM15 disintegrin domain expression level by releasing the rare codons and amino acids restriction. Appl Biochem Biotechnol 157: 299-310, 2009.
17. Hou Y, Chu M, Du FF, et al: Recombinant disintegrin domain of ADAM15 inhibits the proliferation and migration of Bel-7402 cells. Biochem Biophys Res Commun 435: 640-645, 2013.

18. Halpern ME, Thisse C, Ho RK, et al: Cell-autonomous shift from axial to paraxial mesodermal development in zebrafish floating head mutants. Development 121: 4257-4264, 1995.

19. Chang YM, Bai L, Liu S, Yang JC, Kung HJ and Evans CP: Src family kinase oncogenic potential and pathways in prostate cancer as revealed by AZD0530. Oncogene 27: 6365-6375, 2008.

20. Kuefer R, Day KC, Kleer CG, et al: ADAM15 disintegrin is associated with aggressive prostate and breast cancer disease. Neoplasia 8: 319-329, 2006.

21. Bridges LC, Sheppard D and Bowditch RD: ADAM disintegrin-like domain recognition by the lymphocyte integrins alpha4beta1 and alpha4beta7. Biochem J 387: 101-108, 2005.

22. Beck V, Herold H, Benge A, et al: ADAM15 decreases integrin alphavbeta3/vitronectin-mediated ovarian cancer cell adhesion and motility in an RGD-dependent fashion. Int J Biochem Cell Biol 37: 590-603, 2005

23. Jain RK, Duda DG, Clark JW and Loeffler JS: Lessons from phase III clinical trials on anti-VEGF therapy for cancer. Nat Clin Pract Oncol 3: 24-40, 2006.

24. Ferrara N: VEGF-A: a critical regulator of blood vessel growth. Eur Cytokine Netw 20: 158-163, 2009.

25. Carmeliet P and Jain RK: Molecular mechanisms and clinical applications of angiogenesis. Nature 473: 298-307, 2011.

26. Drake CJ, Cheresh DA and Little CD: An antagonist of integrin alpha $\mathrm{v}$ beta 3 prevents maturation of blood vessels during embryonic neovascularization. J Cell Sci 108: 2655-2661, 1995.

27. Hammes HP, Brownlee M, Jonczyk A, Sutter A and Preissner KT: Subcutaneous injection of a cyclic peptide antagonist of vitronectin receptor-type integrins inhibits retinal neovascularization. Nat Med 2: 529-533, 1996.

28. Kerr JS, Wexler RS, Mousa SA, et al: Novel small molecule alpha $\mathrm{v}$ integrin antagonists: comparative anti-cancer efficacy with known angiogenesis inhibitors. Anticancer Res 19: 959-968, 1999.

29. Patel SR, Jenkins J, Papadopolous N, et al: Pilot study of vitaxin - an angiogenesis inhibitor-in patients with advanced leiomyosarcomas. Cancer 92: 1347-1348, 2001

30. Cheng J, Gu YJ, Wang Y, Cheng SH and Wong WT: Nanotherapeutics in angiogenesis: synthesis and in vivo assessment of drug efficacy and biocompatibility in zebrafish embryos. Int J Nanomedicine 6: 2007-2021, 2011.

31. Sofia Vala I, Martins LR, Imaizumi N, et al: Low doses of ionizing radiation promote tumor growth and metastasis by enhancing angiogenesis. PLoS One 5: e11222, 2010.

32. Serbedzija GN, Flynn E and Willett CE: Zebrafish angiogenesis: a new model for drug screening. Angiogenesis 3: 353-359, 1999.

33. Crociani O, Zanieri F, Pillozzi S, et al: hERG1 channels modulate integrin signaling to trigger angiogenesis and tumor progression in colorectal cancer. Sci Rep 3: 3308, 2013.

34. Lucena SE, Romo K, Suntravat $M$ and Sanchez EE: Anti-angiogenic activities of two recombinant disintegrins derived from the Mohave and Prairie rattlesnakes. Toxicon 78 : $10-17,2014$ 